\title{
Efficiency Statistics at All Times: Carnot Limit at Finite Power
}

\author{
M. Polettini, ${ }^{*}$ G. Verley, ${ }^{\dagger}$ and M. Esposito \\ Complex Systems and Statistical Mechanics, Physics and Materials Research Unit, University of Luxembourg, \\ 162 a Avenue de la Faïencerie, L-1511 Luxembourg, Luxembourg
}

(Received 16 September 2014; published 3 February 2015)

\begin{abstract}
We derive the statistics of the efficiency under the assumption that thermodynamic fluxes fluctuate with normal law, parametrizing it in terms of time, macroscopic efficiency, and a coupling parameter $\zeta$. It has a peculiar behavior: no moments, one sub-, and one super-Carnot maxima corresponding to reverse operating regimes (engine or pump), the most probable efficiency decreasing in time. The limit $\zeta \rightarrow 0$ where the Carnot bound can be saturated gives rise to two extreme situations, one where the machine works at its macroscopic efficiency, with Carnot limit corresponding to no entropy production, and one where for a transient time scaling like $1 / \zeta$ microscopic fluctuations are enhanced in such a way that the most probable efficiency approaches the Carnot limit at finite entropy production.
\end{abstract}

DOI: 10.1103/PhysRevLett.114.050601

PACS numbers: 05.70.Ln, 05.70.Fh, 88.05.Bc

Efficiency quantifies how worth a local gain at the expense of a global loss is. In thermodynamics, "losses" are measured by the rate $\bar{\sigma}_{2}>0$ at which entropy is externalized to the environment in the form of a degraded form of energy, while "gain" is the rate $-\bar{\sigma}_{1}$ at which entropy is expelled from a system to upgrade its own state. Globally, entropy is produced at rate $\bar{\sigma}=\bar{\sigma}_{2}+\bar{\sigma}_{1}$, and the second law of thermodynamics $\bar{\sigma} \geq 0$ conveys that locally one cannot earn more of what is globally lost. Then, the efficiency $\bar{\eta}=-\bar{\sigma}_{1} / \bar{\sigma}_{2}$ is bounded by the (scaled) Carnot efficiency $\eta_{c}=1$. Alas, in craving this limit one is deluded by the fact that it occurs at zero power, which is useless for any activity to be accomplished in a reasonable time.

This picture is only tenable for macroscopic systems. For microscopic systems subject to random fluctuations, the concept of a stochastic efficiency has been recently introduced by Verley et al. [1,2]. The first notion one has to revise is that a fluctuating efficiency can indeed exceed the Carnot limit, when in a machine designed to convert in average a form of input power into a form of output power (e.g., an engine producing work at the expense of a heat flow), for a rare event the input and output are reversed (e.g., a pump that employs mechanical work to absorb heat). Moreover, it has been observed that for time-symmetric protocols in the long time limit the Carnot efficiency becomes the least probable in a "large deviation" sense [3] - a very counterintuitive and fascinating result that, in its time-asymmetric variant $[2,4]$, is already subject to experimental inquiry [5]. Corrections at long finite times have been estimated in Ref. [4].

In this Letter, we derive the full probability density function (PDF) of the efficiency, under the assumption that thermodynamic fluxes are distributed with a multivariate Gaussian with cumulants growing linearly in time. The efficiency PDF displays quite peculiar features. In particular, it does not afford moments of any order so that there is no average efficiency and mean-square error. Experimentally, this implies that any data analysis should focus on most probable values. About the latter, after an initial transient the distribution becomes bimodal, as observed numerically in Ref. [6]. As time elapses, the more pronounced maximum drifts towards the always smaller macroscopic value of the efficiency, while a less pronounced maximum at higher efficiency moves in the super-Carnot region towards infinity. We provide a clear physical interpretation of these two peaks. Finally, we argue that the macroscopic framework fails to capture another way of approaching Carnot efficiency at finite entropy production, at finite time, when microscopic fluctuations are enhanced so as to affect the macroscopic behavior.

Macroscopic nonequilibrium thermodynamics [7] is rooted on two assumptions, both of which are today being challenged in the framework of the stochastic theory of nonequilibrium thermodynamics [8,9]: Certain fluxes $\boldsymbol{x}=\left(x_{1}, x_{2}\right)$, with units of an extensive physical quantity per time, take definite values $\bar{x}$. Fluxes are linearly related to their conjugate thermodynamic forces $f$ via $\bar{x}=L f$, where the linear response matrix $L$ is assumed to be positive semidefinite and symmetric by virtue of the Onsager reciprocity relations, yielding a non-negative macroscopic entropy production rate $\bar{\sigma}=f \cdot L f$.

We relax the first assumption, by supposing that at a given time $t$ fluxes $\boldsymbol{x}$ are distributed with law $P_{t}(\boldsymbol{x})$. Each current produces entropy at rate $\sigma_{i}=f_{i} x_{i}$, for a total entropy production rate $\sigma=\sigma_{1}+\sigma_{2}$, with units of $k_{B}$ per time. Then, the adimensional efficiency

$$
\eta=-\frac{f_{1} x_{1}}{f_{2} x_{2}}=-\frac{\sigma_{1}}{\sigma_{2}}
$$

is a stochastic variable distributed with PDF 


$$
\begin{aligned}
P_{t}(\eta) & =\int d x_{1} d x_{2} \delta\left(\eta+\frac{x_{1} f_{1}}{x_{2} f_{2}}\right) P_{t}\left(x_{1}, x_{2}\right) \\
& =\varphi \int d x|x| P_{t}(-\varphi \eta x, x),
\end{aligned}
$$

where $\varphi=f_{2} / f_{1}$ can be assumed to be positive. A remarkable fact one immediately encounters is that the efficiency can fluctuate beyond the Carnot limit. The probability of an efficiency higher than that of Carnot coincides with the probability of negative entropy production rate,

$$
\begin{aligned}
& P_{t}(\eta<1)=P_{t}(\sigma>0)=\langle\theta(\sigma)\rangle_{t}, \\
& P_{t}(\eta>1)=P_{t}(\sigma<0)=\left\langle\theta(\sigma) e^{-t \sigma}\right\rangle_{t},
\end{aligned}
$$

where $\theta$ is Heaviside's step function. The rightmost equations follow from the fluctuation theorem $[10,11]$

$$
\frac{P_{t}(\sigma)}{P_{t}(-\sigma)}=e^{t \sigma}
$$

which states that processes producing negative entropy are exponentially disfavored with respect to those producing positive entropy. Therefore, that super-Carnot efficiencies are unlikely compared to sub-Carnot efficiencies is an incarnation of the fluctuation theorem.

Exact results can be obtained by assuming that fluxes are distributed with normal multivariate density function

$$
P_{t}(\boldsymbol{x})=\frac{t}{4 \pi \sqrt{|L|}} \exp \left[-\frac{t}{4}(\boldsymbol{x}-\overline{\boldsymbol{x}}) \cdot L^{-1}(\boldsymbol{x}-\overline{\boldsymbol{x}})\right],
$$

where $|\cdot|$ is the determinant. That (one-half) the correlation matrix should be identified with the linear response matrix is corroborated by the Green-Kubo relations

$$
L_{i j}=\frac{t}{2}\left\langle\left(x_{i}-\bar{x}_{i}\right)\left(x_{j}-\bar{x}_{j}\right)\right\rangle,
$$

another well-known consequence of the fluctuation theorem [12]. The time dependence in Eq. (5) is due to the fact that the time-integrated fluxes $t \overline{\boldsymbol{x}}$ increase linearly in time, and correspondingly so do their cumulants. Under these assumptions, the efficiency PDF Eq. (2) can be exactly calculated (see the Supplemental Material [13]). It only depends on four adimensional parameters: The macroscopic efficiency $\bar{\eta}$, the coupling parameter $\zeta=$ $|L| /\left(L_{11} L_{22}\right) \in[0,1]$ that for thermoelectric devices [14] is related to the so-called figure of merit $z T=1 / \zeta-1$, the average entropy production rate $\bar{\sigma}$, which sets the time scale and can be reabsorbed by a time reparametrization $\tau=t \bar{\sigma}$, and $\epsilon= \pm 1$. With $\bar{\sigma}$ being the only extensive parameter, large $\tau$ stands both for large times and the macroscopic limit. We obtain (Supplemental Material [13])

$$
P_{\tau}(\eta)=\frac{e^{-\tau / 4}}{\pi a(\eta) \sqrt{|C|}}\left\{1+\sqrt{\pi \tau} h(\eta) e^{\tau h(\eta)^{2}} \operatorname{erf}[\sqrt{\tau} h(\eta)]\right\}
$$

where erf is the error function and

$$
\begin{aligned}
& a(\eta)=(1-\eta)^{2}+\frac{1}{|C|}\left(\frac{\eta-\bar{\eta}}{1-\bar{\eta}}\right)^{2}, \\
& h(\eta)=\frac{1-\eta}{2 \sqrt{a(\eta)}} .
\end{aligned}
$$

Here, $|C|=|L| f_{1}^{2} f_{2}^{2} / \bar{\sigma}^{2}$ is the determinant of the matrix with dimensionless entries $C_{i j}=L_{i j} f_{i} f_{j} / \bar{\sigma}$. It can be expressed in terms of our parameters as

$$
|C|=\frac{z T}{2}\left(1+\epsilon \sqrt{1-\frac{4}{z T} \frac{\bar{\eta}}{(1-\bar{\eta})^{2}}}\right)-\frac{\bar{\eta}}{(1-\bar{\eta})^{2}},
$$

where $\epsilon= \pm$ accounts for the existence of two probability distributions corresponding to given parameters. For $|L|$ to be real, the known bound

$$
\bar{\eta} \leq \frac{1-\sqrt{\zeta}}{1+\sqrt{\zeta}}
$$

must hold [14]. Importantly, $a(\eta)$ is positive semidefinite.

Let us study the efficiency PDF in detail. First, it is a power-law distribution with tails

$$
P_{\tau}(\eta \rightarrow \pm \infty) \propto \eta^{-2},
$$

which, after submission of this Letter, has been proven to be a universal property of efficiency distributions [15]. As a consequence, it does not afford finite moments of any order. Hence, the macroscopic efficiency $\bar{\eta}$ is not the average efficiency $\langle\eta\rangle_{t}$, which is not finite.

In Fig. 1, the efficiency distribution is plotted as the bold curve. Remarkably, for a large class of parameters it displays two maxima at $\eta_{m}, \eta_{m}^{*}$ and a minimum, the latter slightly off the Carnot efficiency. Hence, not only superCarnot efficiencies are possible, but indeed, there appears a local maximum with an efficiency higher than that of Carnot. To understand its physical origin, we distinguish four operational regimes of the machine, according to the signs of the two contributions $\sigma_{1}$ and $\sigma_{2}$ to the entropy production rate. The two regimes contributing to positive efficiencies are the machine -+ that employs process 2 flowing along its spontaneous tendency, to drive process 1 against its spontaneous tendency (e.g., heat engine) and the dual machine +- where the system's spontaneous tendency is used to drive the environment against its tendency (e.g., the heat pump). Correspondingly, we have $\theta(\eta) P_{\tau}(\eta)=P_{\tau}^{+-}(\eta)+P_{\tau}^{-+}(\eta)$ where 


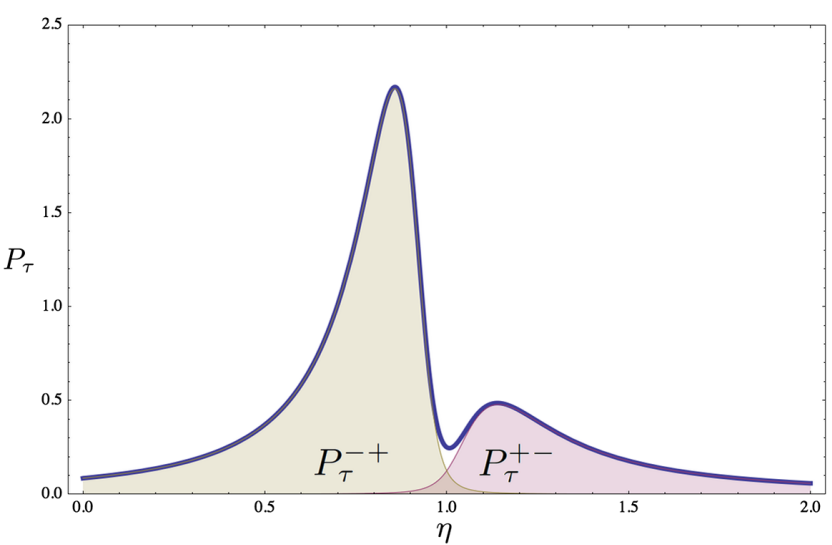

FIG. 1 (color online). Bold curve: efficiency distribution $P_{\tau}(\eta)$ for parameter values $\zeta=0.01, \bar{\eta}=0.6, \tau=10, \epsilon=+1$. Filled curves beneath: $P_{\tau}^{-+}(\eta)$ and $P_{\tau}^{+-}(\eta)$, showing that each maximum is mostly due to one working mode of the engine.

$$
P_{\tau}^{+-}(\eta)=\int_{\substack{+\sigma_{1}>0 \\-\sigma>0}} d x_{1} d x_{2} P_{t}\left(x_{1}, x_{2}\right) \delta\left(\eta+\frac{f_{1} x_{1}}{f_{2} x_{2}}\right)
$$

and similarly for $P_{\tau}^{-+}$. Shaded plots are provided in Fig. 1, showing that each of the two maxima is almost exclusively determined by one of the two modes of the machine, the second of which by inversion of input and output has typical efficiency $1 / \eta_{m}^{*}<1$. Regimes ++ and -- contribute to the tail of the distribution at $\eta<0$.

Let us now study the behavior of $P_{\tau}(\eta)$ in scaled time, depicted in Fig. 2. At $\tau=0$ we obtain a Cauchy distribution $P_{0}(\eta)=1 /[\pi a(\eta) \sqrt{|C|}]$, with maximum at $\eta_{0}=-L_{12} f_{1} /\left(L_{22} f_{2}\right)$. We have $\eta_{0} \geq \bar{\eta}$, and equality can only occur for $|C|=0$. This implies that the most probable efficiency decreases in time towards $\bar{\eta}$. Furthermore, at thermodynamic equilibrium where all the forces vanish,

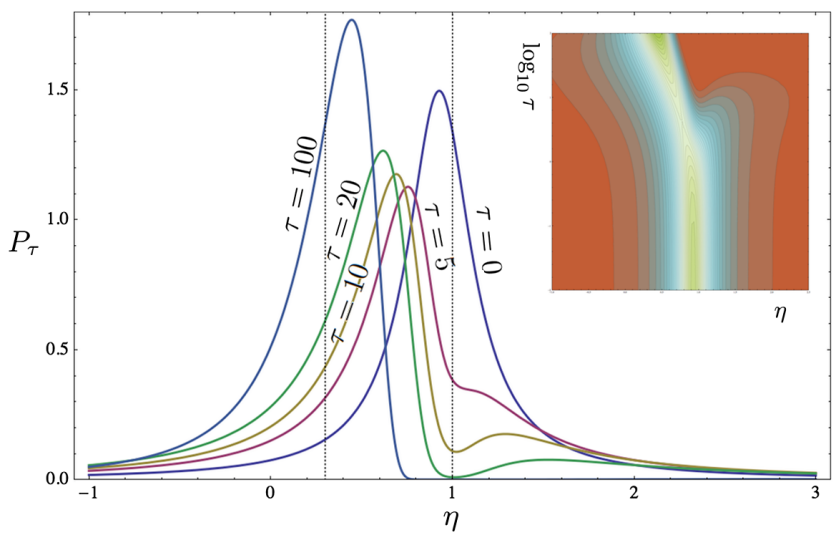

FIG. 2 (color online). Main frame: Efficiency distribution at various scaled times, for $\zeta=0.05, \bar{\eta}=0.3, \epsilon=+$. The vertical dotted lines correspond to $\bar{\eta}$ and $\eta_{c}$. Inset: Contour plot of the efficiency PDF as a function of $\eta$ and $\tau$ (in log scale). Maxima are points where the level lines have horizontal tangents. After a critical time, a second maximum drifting to infinity appears. $\boldsymbol{f} \rightarrow 0$ at finite $\varphi$, it can be shown that $P_{\tau}^{\mathrm{eq}}(\eta)=P_{0}(\eta)$, which means that systems at equilibrium do not evolve. As time elapses a transition to a bimodal distribution occurs, with the super-Carnot maximum drifting to infinity. We can define a critical time $\tau_{c}$ at which there appears an inflection point in $P_{\tau}(\eta)$. Numerical plots of $\tau_{c}$ in terms of $\bar{\eta}$ and $c$ show that the critical time is higher the closer to the maximal efficiency and to the "loose coupling" condition $\zeta \rightarrow 1$ (Supplemental Material [13]). Finally, in the long time limit one has $\operatorname{erf}(\sqrt{\tau} h) \sim 1-e^{-\tau h^{2}} /(\sqrt{\pi \tau}|h|)[16]$ and

$$
P_{\tau \rightarrow \infty}(\eta) \sim \frac{e^{-\tau / 4}}{\pi a(\eta)|C|}\left(1-\frac{h}{|h|}+\sqrt{\pi \tau} e^{\tau h(\eta)^{2}}\right) .
$$

The large-time behavior is captured by the large deviation rate function $I(\eta)=-\lim _{\tau \rightarrow \infty} \tau^{-1} \ln P_{\tau}(\eta)=$ $1 / 4-h(\eta)^{2} \geq 0$, which was first calculated and thoroughly analyzed by Verley et al. [1,2]. The rate function has only two extrema, a minimum $I(\bar{\eta})=0$ and a maximum $I(1)=1 / 4$, and asymptotically $I( \pm \infty)=\left[4|C|(1-\bar{\eta})^{2}+\right.$ $4]^{-1} \leq I(1)$. Then, the more pronounced maximum tends to the macroscopic efficiency $\bar{\eta}$, while the minimum tends to the Carnot efficiency. The second maximum does not appear in the large deviation rate function because at infinite time it moves to infinity, since it belongs to a subdominant decay mode. This proves the existence of a critical time $\tau_{c}$, as there must exist another maximum for the distribution to converge.

The quest for the Carnot limit is very subtle. By Eq. (10), the Carnot bound can be saturated in the limit $\zeta \rightarrow 0$, giving rise to two extreme situations related to the spectrum and eigenvectors of the response matrix $L \rightarrow L^{\epsilon}$. For $\epsilon=-$ ("tight coupling"), by Eq. (9) the correlation matrix becomes degenerate,

$$
L^{-}=\left(\begin{array}{cc}
L_{11} & -\sqrt{L_{11} L_{22}}+O(\zeta) \\
-\sqrt{L_{11} L_{22}}+O(\zeta) & L_{22}
\end{array}\right),
$$

where $O(\zeta)$ are terms of order $\zeta$. For $\epsilon=+$ ("singular coupling"), $L$ tends to the inverse of a degenerate matrix, i.e., $L^{+}=L^{-} / O(\zeta)$, with $\left|L^{+}\right| \rightarrow \infty$.

To reach Carnot efficiency, a second independent condition ("self-duality") must hold: $\varphi$ attains value $\varphi^{*}=\sqrt{L_{11} / L_{22}}$, which affords an interesting interpretation in terms of the probability of the inverse efficiency (Supplemental Material [13]). When $\zeta \rightarrow 0$, this condition makes $\boldsymbol{f}$ either the null eigenvector of $L^{-}$relative to its null eigenvalue or of $L^{+}$relative to its finite eigenvalue. In the tight-coupling regime, this condition is known as the "stall force" [17].

Expressing the efficiency in terms of the adimensional parameters $\zeta$ and $\phi=\varphi / \varphi^{*}$ (for $L_{12}<0$ ) as [18]

$$
\bar{\eta}(\zeta, \phi)=-\frac{1-\phi \sqrt{1-\zeta}}{\phi^{2}-\phi \sqrt{1-\zeta}},
$$


one finds that the two limits towards self-duality and towards tight or singular coupling do not commute,

$$
1=-\lim _{\zeta \rightarrow 0} \lim _{\phi \rightarrow 1} \bar{\eta}=+\lim _{\phi \rightarrow 1} \lim _{\zeta \rightarrow 0} \bar{\eta} .
$$

Then, a macroscopic Carnot efficiency is "fragile," as the self-dual forces needed to attain it are those that slightly out of $\zeta=0$ give a "dud" machine that dissipates to obtain nothing, with macroscopic efficiency $\bar{\eta}=-1$.

Nevertheless, the probabilistic level is richer. At tight coupling the bivariate Gaussian Eq. (5) becomes univariate with support along $x_{1} / x_{2}=-\varphi^{*}$, and the efficiency PDF a Dirac delta centered at the macroscopic efficiency. Then tightly coupled machines work macroscopically at all scaled times.

More interesting is the singular coupling. Figure 3 shows that in this limit all extrema tend to accumulate towards the Carnot efficiency, where the density concentrates. Despite the fact that the two peaks survive, convergence to a Dirac delta can be proven by the following argument [19]: From Eqs. (7) and (8), $h \rightarrow 1 / 2, a \rightarrow(1-\eta)^{2}$, and the efficiency PDF converges to a distribution with support in $\eta=1$, which is then necessarily a finite combination of derivatives of the Dirac delta $P_{\tau}(\eta)=\sum_{n=0}^{N} p_{n} \delta^{(n)}(1-\eta)$ [20]. Since $\langle g\rangle>0$ for all positive test functions $g(\eta)>0$, then necessarily $p_{n}=0$ but for $p_{0}=1 \square$. Then, singular coupling pushes the most probable efficiency towards the Carnot limit at fixed $\tau$; the shadings in Fig. 3 suggest that in this limit the distribution is fairly insensitive to $\bar{\eta}$. Moreover, the contour plot in Fig. 3 supports that the most probable efficiency stays at the same value for probability densities evaluated at a fixed time $\tau \propto 1 / \zeta$, showing that

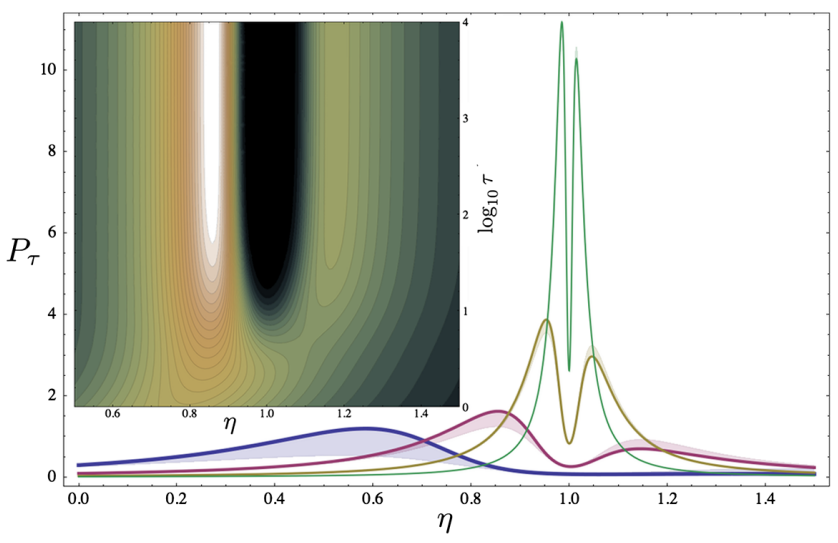

FIG. 3 (color online). Graphs of $\left(\eta, P_{\tau}(\eta)\right)$ for $\tau=10, \bar{\eta}=0.3$, $\epsilon=+$ and for various coupling parameters (from bolder to thinner) $\zeta=0.1,0.01,0.001,0.0001$. The shading represents the distance to the corresponding curves for $\bar{\eta}=-1$. Inset: Contour plot of the efficiency PDFs corresponding to parameter $\zeta=0.1 / \tau$ as a function of the efficiency $\eta$ and the scaled time $\tau$ (in $\log$ scale), showing that the PDF is invariant at all times, hence, that singular coupling stretches the relaxation times. Lighter tones for higher probabilities, darker for lower. convergence to $\bar{\eta}$ is more and more delayed. However, it must be remembered that the physical time scale is set by the entropy production rate. Necessarily, the matrix entries of $L^{+}$diverge; then in general $\bar{\sigma}$ also diverges. Still, $L^{+}$ admits a finite eigenvalue. Picking the forces along the relative eigenvector, $\phi=1+O(\zeta)$, one obtains a finite entropy production rate. Oddly, as discussed above, these conditions are met when the macroscopic machine is dud.

To resume: At singular coupling, the effect of fluctuations is macroscopically visible and permits us to work close to Carnot efficiency at finite entropy production rate for sufficiently long physical times. The conditions for which the entropy production rate can be held finite are those under which the machine eventually evolves towards a dud fate. Notice that in this regime the system might flip randomly across the close sharp peaks of the PDF. However, the inset in Fig. 3 suggests that at intermediate times reasonably high typical efficiencies will be favored and that a large separation between such peaks (the dark region of zero probability) occurs. Hence, to put it with a motto, a singular machine doomed to be useless might be efficiently useful for some time due to fluctuations; the better in the short run, the worse in the long. By the GreenKubo relation of Eq. (6) the singular coupling limit is approached when correlations between the currents diverge and the inverse correlation matrix becomes degenerate. It is tempting to parallel this behavior to the paradigm of criticality at phase transitions, where fluctuations become macroscopic, correlations diverge, and the covariance matrix degenerates $[21,22]$.

An important observation to be made here is that singular coupling pushes the system far from equilibrium. The framework of stochastic thermodynamics encompasses such systems by assuming that they are subtended by an underlying Markovian dynamics, giving rise to nonGaussian current statistics. Gaussianity is only recovered in the linear regime at large times by the central limit theorem [23,24]. While the model of a Brownian particle in a tilted plane studied in Ref. [1] has the exact Gaussian propagators studied in this Letter, in general Markov processes have a more complex behavior in time; in particular, the average flux varies as the system evolves, depending on the initial ensemble. Then, the exact shortand large-time behavior of the efficiency distribution might become model dependent. For asymmetric protocols, a signature of non-Gaussian behavior is the off-Carnot leastprobable efficiency $[2,4,5]$.

Nevertheless, our study points out that in the simplest Gaussian scenario the efficiency PDF manifests peculiar features that might possibly be universal: power-law tails, no finite moments, a naturally occurring transition to a bimodal distribution due to reverse working regimes, etc. Particularly intriguing is the limit of a degenerate or singular covariance matrix. While the former case is intrinsically macroscopic and broadly studied [14,18], 
we obtain a clear indication that the singular coupling regime displays an interesting behavior that could lead to the enhancement of the efficiency above its macroscopic value. More light is to be shed on these issues by future inquiry on the finite-time statistics of the efficiency in stochastic models $[15,17]$ in their rich phenomenology, including maximum power generation $[25,26]$, multiterminal machines [27], broken time-reversal symmetry [28], the insurgence of phase transitions, and in relation to the issue of efficiency enhancement by noise [29] or by decoherence [30]. Experimental setups that could test these predictions are already available [31-35]. The full statistics of the efficiency close to equilibrium has recently been sampled for a Carnot engine realized with a Brownian particle, in the quasistatic limit where the currents' statistics is Gaussian [5], and data analysis farther away from equilibrium might soon be available.

This research was supported by the National Research Fund Luxembourg in the frame of project FNR/A11/02 and of Postdoc Grant No. 5856127.

*matteo.polettini@uni.lu

† gatien.verley@th.u-psud.fr

\#massimilano.esposito@uni.lu

[1] G. Verley, T. Willaert, C. Van den Broeck, and M. Esposito, Nat. Commun. 5, 4721 (2014).

[2] G. Verley, T. Willaert, C. Van den Broeck, and M. Esposito, Phys. Rev. E 90, 052145 (2014).

[3] H. Touchette, Phys. Rep. 478, 1 (2009).

[4] T. R. Gingrich, G. M. Rotskoff, S. Vaikuntanathan, and P. L. Geissler, New J. Phys. 16, 102003 (2014).

[5] I. A. Martinez, E. Roldan, L. Dinis, D. Petrov, J. M. R. Parrondo, and R. Rica, arXiv:1412.1282.

[6] S. Rana, P. S. Pal, A. Saha, and A. M. Jayannavar, Phys. Rev. E 90, 042146 (2014).

[7] S. R. De Groot and P. Mazur, Non-equilibrium Thermodynamics (Courier Dover Publications, New York, 2013).

[8] U. Seifert, Rep. Prog. Phys. 75, 126001 (2012).

[9] C. Van den Broeck and M. Esposito, Physica (Amsterdam) 418A, 6 (2015).

[10] G. N. Bochkov and Y. E. Kuzovlev, Physica (Amsterdam) 106A, 443 (1981); G. N. Bochkov and Y.E. Kuzovlev, Physica (Amsterdam) 106A, 480 (1981).

[11] M. Polettini and M. Esposito, J. Stat. Mech., (2014) P10033.

[12] D. Andrieux and P. Gaspard, J. Chem. Phys. 121, 6167 (2004).
[13] See the Supplemental Material at http://link.aps.org/ supplemental/10.1103/PhysRevLett.114.050601 for details regarding the derivation of the efficiency PDF, the parametrization, and the various limiting situations.

[14] G. Benenti, K. Saito, and G. Casati, Phys. Rev. Lett. 106, 230602 (2011).

[15] K. Proesmans, B. Cleuren, and C. Van Den Broeck, arXiv:1411.3531.

[16] M. Abramowitz and I. A. Stegun, Handbook of Mathematical Functions with Formulas, Graphs, and Mathematical Tables (Dover, New York, 1965).

[17] F. Jülicher, A. Ajdar, and J. Prost, Rev. Mod. Phys. 69, 1269 (1997).

[18] O. Entin-Wohlman, J.-H. Jiang, and Y. Imry, Phys. Rev. E 89, 012123 (2014).

[19] Discussion with user Kostya_I on mathoverflow.net, http:// mathoverflow.net/questions/178859/ power-law-distribution-with-support-in-x-0.

[20] L. Hörmander, The Analysis of Linear Partial Differential Operators I (Springer, Berlin, 1983).

[21] P. Zanardi, P. Giorda, and M. Cozzini, Phys. Rev. Lett. 99 , 100603 (2007).

[22] M. Polettini, Eur. Phys. J. B 87, 215 (2014).

[23] T. Speck and U. Seifert, Phys. Rev. E 70, 066112 (2004).

[24] J. Hoppenau and A. Engel, J. Stat. Mech.: Theory Exp. (2013) P06004.

[25] M. Esposito, K. Lindenberg, and C. Van den Broeck, Phys. Rev. Lett. 102, 130602 (2009).

[26] T. Schmiedl and U. Seifert, Europhys. Lett. 81, 20003 (2008).

[27] F. Mazza, R. Bosisio, G. Benenti, V. Giovannetti, R. Fazio, and F. Taddei, New J. Phys. 16, 085001 (2014).

[28] K. Saito, G. Benenti, G. Casati, and T. Prosen, Phys. Rev. B 84, 201306 (2011).

[29] J. Spiechowicz, P. Hänggi, and J. Łuczka, Phys. Rev. E 90, 032104 (2014).

[30] F. Caruso, A. W. Chin, A. Datta, S. F. Huelga, and M. B. Plenio, J. Chem. Phys. 131, 105106 (2009).

[31] D. Collin, F. Ritort, C. Jarzynski, S. B. Smith, I. Tinoco, and C. Bustamante, Nature (London) 437, 231 (2005).

[32] A. Bérut, A. Arakelyan, A. Petrosyan, S. Ciliberto, R. Dillenschneider, and E. Lutz, Nature (London) 483, 187 (2012).

[33] J. V. Koski, T. Sagawa, O-P. Saira, Y. Yoon, A. Kutvonen, P. Solinas, M. Möttönen, T. Ala-Nissila, and J. P. Pekola, Nat. Phys. 9, 644 (2013).

[34] S. Ciliberto, A. Imparato, A. Naert, and M. Tanase, Phys. Rev. Lett. 110, 180601 (2013).

[35] C. Tietz, S. Schuler, T. Speck, U. Seifert, and J. Wrachtrup, Phys. Rev. Lett. 97, 050602 (2006). 